\section{Conhecimento sobre anticoncepção de emergência entre adolescentes do Ensino Médio de escolas públicas e privadas}

\author{
Knowledge of emergency contraception among \\ adolescents in public and private Brazilian \\ high schools
}

\section{Conocimiento sobre anticoncepción de emergencia en adolescentes de escuela secundaria en escuelas públicas y privadas}

\author{
1 Escola de Enfermagem, \\ Universidade de São Paulo, \\ São Paulo, Brasil. \\ Correspondência \\ C. B. N. Chofakian \\ Escola de Enfermagem, \\ Universidade de São Paulo. \\ Rua Dr. Enéias de Carvalho \\ Aguiar 419, São Paulo, SP \\ 05403-000, Brasil. \\ christianeborges@gmail.com
}

\section{Abstract}

This study aimed to analyze the level of knowledge concerning emergency contraception among adolescents in public and private high schools. This was a cross-sectional study with 705 students 15 to 19 years of age enrolled in public and private high schools in a municipality in São Paulo State, Brazil. The authors used stratified probabilistic sampling by type of school and systematic sampling by class. Sexual initiation and use of emergency contraception were reported by $24.9 \%$ of private school students and $32 \%$ of public school students. The mean score on knowledge was $3.87(S D=2.12)$ in public schools and 5.14 $(S D=2.00)$ in private schools. Multiple linear regression analysis showed that higher scores on knowledge concerning emergency contraception were associated with: enrollment in private schools, female gender, older adolescents, sexual initiation, previous use of emergency contraception, and knowing someone who had used the method. The study concludes that few adolescents are properly informed about the method and that many harbor persistent misconceptions.

Adolescent Health; Postcoital Contraception; Sexual and Reproductive Health
Christiane Borges do Nascimento Chofakian 1 Ana Luiza Vilela Borges 1

Elizabeth Fujimori 1

Luiza Akiko Komura Hoga 1

\section{Resumo}

O objetivo do estudo foi analisar o nivel de conhecimento sobre anticoncepção de emergência entre adolescentes do Ensino Médio de escolas públicas e privadas. Estudo transversal com a participação de 705 estudantes de 15 a 19 anos, matriculados no Ensino Médio de escolas públicas e privadas de um município do Estado de São Paulo, Brasil. Utilizou-se amostragem probabilística estratificada por tipo de escola e sistemática por turma. Cerca de $24,9 \%$ dos adolescentes das escolas privadas e $32 \%$ das escolas públicas haviam iniciado a vida sexual e usado a anticoncepção de emergência. A média de escore de conhecimento foi de $3,87(D P=2,12)$ nas escolas públicas e 5,14 (DP =2,00) nas escolas privadas. Análise de regressão linear múltipla mostrou que adolescentes de escolas privadas, do sexo feminino, com mais idade, que já tinham iniciado a vida sexual, usado a anticoncepção de emergência e conheciam alguém que já usou o método, foram as que alcançaram o maior escore de conhecimento. Conclui-se que poucos adolescentes estão corretamente informados sobre o método e muitos têm ideias equivocadas.

Saúde do Adolescente; Anticoncepção Pós-Coito; Saúde Sexual e Reprodutiva 
Introdução

A Organização Mundial da Saúde define anticoncepção de emergência como método anticoncepcional (MAC) a ser usado após a relação sexual para prevenir a gravidez ${ }^{1}$. Seu uso é indicado para mulheres em idade reprodutiva, incluindo as adolescentes, após relações sexuais desprotegidas, que resultaram de falhas, uso incorreto de métodos contraceptivos de uso regular, ou em caso de agressão sexual 2. As adolescentes representam um grupo importante para a ampliação do acesso a todas as formas de contracepção, incluindo anticoncepção de emergência, tendo em vista que o início da atividade sexual geralmente ocorre durante esta fase do curso da vida 3 e suas práticas contraceptivas são caracterizadas pela descontinuidade e ocorrência de falhas ${ }^{4}$.

Os resultados da Pesquisa Nacional de Demografia e Saúde (PNDS), realizada em 2006, mostraram que a maioria (69\%) das adolescentes brasileiras já ouviu falar na anticoncepção de emergência 5. No entanto, a citação espontânea de um MAC não significa necessariamente que a pessoa conhece o método, ou seja, que ela possua informações suficientes sobre as vantagens, desvantagens, formas de acesso e o modo de usálo 6 . Estudos que avaliaram o nível do conhecimento sobre anticoncepção de emergência entre adolescentes e jovens revelaram que eles pouco conhecem a respeito das indicações, mecanismos de ação ou a eficácia dos MACs 7,8.

Diante da importância que o uso dos MACs, em especial a anticoncepção de emergência, adquire no perfil de saúde sexual e reprodutiva dos adolescentes, associado ao fato do nível de conhecimento poder interferir positiva ou negativamente no uso 9 da anticoncepção de emergência, desenvolveu-se o presente trabalho, que teve como objetivo analisar o nível de conhecimento da anticoncepção de emergência entre adolescentes de 15 a 19 anos de idade, estudantes do Ensino Médio de escolas públicas e privadas. Considerando-se que o nível de conhecimento sobre a anticoncepção de emergência pode variar em função da idade, da experiência sexual e da inserção social dos adolescentes 10,11, partimos da hipótese que o tipo de escola frequentada pelo adolescente (pública ou privada) está associado ao nível de conhecimento sobre a anticoncepção de emergência, conforme evidenciam outros estudos que focalizaram diferentes MACs 12,13. Nesse contexto, o nível socioeconômico foi medido por meio da estratificação da amostra em escolas públicas e privadas.

\section{Metodologia}

Os dados deste artigo foram extraídos de um projeto de investigação mais ampla, que analisou o conhecimento e uso da anticoncepção de emergência entre adolescentes de escolas públicas e privadas de um município de médio porte da Região Metropolitana de São Paulo, Brasil. Esse estudo transversal foi desenvolvido com adolescentes solteiros de ambos os sexos, regularmente matriculados em escolas de Ensino Médio da região urbana. Cabe destacar que foram considerados apenas os adolescentes do período matutino, pois no período noturno a maioria tinha idades entre 18 e 22 anos, segundo informação da Diretoria de Ensino. O critério de inclusão adotado foi o relato do conhecimento sobre anticoncepção de emergência, e os critérios de exclusão foram os adolescentes com união formal ou informal e aqueles com menos de 15 anos de idade.

Dados municipais fornecidos pela Diretoria de Ensino, indicaram que em 2011 havia 2.507 estudantes regularmente matriculados no Ensino Médio, distribuídos em quatorze escolas públicas e seis privadas. Para o cálculo amostral, considerou-se que $30,9 \%$ de adolescentes de 15 a 19 anos de idade da Região Sudeste já utilizaram a anticoncepção de emergência 11, com erro máximo em valor absoluto de $4 \%$, nível de 95\% de confiança e fator de correção para população finita. Optou-se por utilizar a variável "uso da anticoncepção de emergência” como parâmetro para o cálculo amostral, uma vez que o uso remete, obrigatoriamente, a pelo menos ter ouvido falar sobre o método. O cálculo amostral indicou a necessidade de 669 adolescentes, ao qual foram acrescidos $20 \%$ para que não houvesse redução decorrente de perdas, o que resultou na amostra final de 803 adolescentes.

Para selecioná-los utilizou-se o método de amostragem por conglomerado, realizado em múltiplos estágios. No primeiro momento, a população foi dividida em dois estratos de acordo com o tipo de escola, tendo sido $2.062(82,2 \%)$ adolescentes de escolas públicas e 445 (17,8\%) de escolas privadas. A estratificação foi realizada com probabilidade proporcional ao tamanho das redes pública e privada. Dessa forma, concluiuse que seriam necessários 660 adolescentes das escolas públicas e 143 das privadas. No segundo momento, procedeu-se à amostragem sistemática das turmas organizadas por ordem alfabética das escolas, sem reposição. Considerando que havia uma média de 37 alunos por turma nas escolas públicas e 23 nas escolas privadas, haveria a necessidade de obter dados junto a 18 turmas de escolas públicas e seis de escolas privadas. To- 
das as escolas foram listadas por ordem alfabética, separadamente por pública e privada, com suas respectivas turmas. Para iniciar a seleção das turmas, foi feito o sorteio de uma dentre as 56 turmas das escolas públicas e uma dentre as 19 turmas das escolas privadas. O intervalo de amostragem foi calculado com base na quantidade de turmas necessárias em cada tipo de escola ( $k=3$, para ambas as escolas). Assim sendo, após o início casual, uma a cada três turmas foi selecionada e, este critério de aleatoriedade foi seguido até se obter a quantidade de turmas necessárias para cada tipo de escola. Dentre as 14 escolas públicas, 18 turmas foram sorteadas em seis escolas diferentes. Das seis escolas privadas, seis turmas foram sorteadas, uma turma em cada escola.

O instrumento de coleta de dados foi um questionário elaborado com base em outros estudos 10,14. Esse questionário foi estruturado, autoaplicado e previamente testado em uma escola, cujo alunos não participaram da pesquisa.

A coleta de dados foi realizada entre agosto e outubro de 2011, após a aprovação do estudo por um Comitê de Ética em Pesquisa credenciado no Conselho Nacional de Ética em Pesquisa (processo no 1056/2011). A autorização para realizar o trabalho foi obtida da Coordenadoria de Ensino do Interior e da Diretoria de Ensino do Município. Inicialmente, contataram-se os coordenadores e diretores das escolas para apresentar o projeto de pesquisa e suas finalidades. Dentre as escolas privadas, três recusaram o convite, duas com a justificativa de que o assunto era inadequado aos adolescentes e outra porque era uma escola com perfil religioso. Dentre as escolas públicas, não houve recusa. Entretanto, nessas escolas havia a problemática do absenteísmo com predomínio nas sextas-feiras. Acresce o fato de que uma das escolas autorizou a aplicação do instrumento apenas as sextas-feiras. Esse fato resultou na participação de uma menor proporção de adolescentes daquela instituição. Optou-se pela inclusão das turmas subsequentes da lista, como uma forma de sanar esse problema. Tomou-se o cuidado de incluir estudantes do mesmo ano escolar com o objetivo de diminuir o viés da aleatoriedade na composição da amostra da população.

Para a coleta de dados, conforme acordado com o corpo docente e a diretoria das escolas, os adolescentes das turmas selecionadas foram convidados a participar do estudo no início de uma aula, quando foi distribuído o Termo de Consentimento Livre e Esclarecido (TCLE) aos alunos, enfatizando-se que aqueles com menos de 18 anos de idade deveriam entregar o TCLE aos pais ou responsáveis, para que os mesmos assinassem, caso aceitassem a sua participação na pesquisa. Os adolescentes com 18 anos de idade ou mais poderiam assinar o TCLE, caso consentissem em participar da pesquisa.

No dia seguinte à entrega do TCLE, os questionários foram distribuídos simultaneamente nas salas de aula para evitar que os alunos compartilhassem os conhecimentos sobre a anticoncepção de emergência. Foi solicitado aos adolescentes que depositassem o instrumento preenchido em uma urna que estava disposta na entrada da sala de aula para esta finalidade. Após o término da pesquisa, conforme acordado com a direção das escolas, foram realizadas oficinas para alunos e professores focalizando a temática abordada.

No total, foram distribuídos 803 TCLE, sendo que 729 adolescentes o entregaram assinado e apenas seis recusaram-se a responder ao questionário. Em conformidade com os critérios de exclusão, a amostra final resultou em 705 adolescentes. Para corresponder ao objetivo deste estudo, foram considerados apenas os alunos que relataram conhecer a anticoncepção de emergência $(n=664)$. Cabe ressaltar que esse número é um pouco menor que o apresentado no cálculo amostral, no entanto, foi baseado no projeto de investigação mais ampla, o qual contemplava análise do uso e do nível de conhecimento da anticoncepção de emergência.

A variável de interesse foi o nível de conhecimento da anticoncepção de emergência. Foram elaboradas dez questões que tinham como alternativas as categorias "verdadeiro", "falso" e "não sei”. O conhecimento a respeito da anticoncepção de emergência foi analisado de duas formas. Primeiro, analisaram-se as respostas corretas, ou seja, a proporção de adolescentes que soube responder corretamente à questão. Posteriormente, elaborou-se um escore de conhecimento, atribuindo-se um ponto para cada questão com resposta correta, de modo que a pontuação total variou de zero (ausência total de acertos) a dez (resposta correta em todas as afirmativas). $\mathrm{Na}$ análise dos escores, as respostas "não sei" foram tratadas como erro, ou seja, receberam pontuação zero.

A principal variável independente foi o tipo de escola (pública/privada). As demais variáveis independentes foram: ano escolar (1o ano/2o ano/3o ano); idade (em anos); sexo (masculino/ feminino); religião (católica/evangélica/espírita/judaica/nenhuma); coabitação/pessoas com quem mora (mãe/pai/mãe e pai/outros); namoro atual (não/sim); início da vida sexual (sim/ não); conhecimento de alguém que já usou a anticoncepção de emergência (não/sim); pessoas ou locais em que o adolescente obteve informa- 
ções sobre a anticoncepção de emergência (pais ou parentes/amigos ou conhecidos/serviços de saúde/revistas ou jornais/televisão/farmácia/ escola/Internet) e uso anterior da anticoncepção de emergência (não/sim/não sabe).

As variáveis início da vida sexual e uso anterior da anticoncepção de emergência foram agrupadas em uma única variável: início da vida sexual/uso anterior da anticoncepção de emergência, de forma que esta variável abrangeu quatro categorias: não iniciou vida sexual e não usou anticoncepção de emergência; iniciou vida sexual, mas não usou anticoncepção de emergência; iniciou vida sexual e usou anticoncepção de emergência; e iniciou vida sexual e não sabe se usou anticoncepção de emergência (para o caso de adolescentes do sexo masculino). Quanto às fontes de informação, foram admitidas respostas múltiplas, sendo estas apresentadas na forma de alternativas.

Todas as análises foram conduzidas no programa estatístico Stata 12.0 (Stata Corp., College Station, Estados Unidos) com o uso de peso amostral pós-estratificação. Foi necessário usar esse recurso, pois embora tenha havido recusas nas escolas privadas e absenteísmo nas sextasfeiras nas escolas públicas, obteve-se maiores proporções de questionários respondidos nas escolas privadas e menores nas públicas, segundo o cálculo amostral proporcional ao tamanho do tipo de escola. Dessa forma, buscou-se compensar a super-representação de respostas oriundas das escolas privadas e a sub-representação de respostas das escolas públicas. Considerando o peso como sendo o inverso da probabilidade de selecionar um sujeito em cada tipo de escola, o peso pós-estratificação resultou da razão entre a frequência relativa de adolescentes entrevistados nas escolas públicas $(76,2 \%)$ e privadas $(23,8 \%) \mathrm{e}$ a população de estudantes matriculados no Ensino Médio segundo dados da Diretoria de Ensino do Município.

A análise descritiva foi realizada por meio de proporção, média e desvio-padrão. As médias do escore de conhecimento foram comparadas usando-se o teste t-Student. A colinearidade foi analisada mediante o coeficiente de correlação Phi para as variáveis categóricas ordinais (ano escolar e idade) e a correlação de Spearman foi utilizada para as variáveis categóricas nominais (namoro atual e início da vida sexual). A análise dos determinantes do nível de conhecimento da anticoncepção de emergência foi realizada utilizando-se a análise da regressão linear múltipla, considerando o escore do conhecimento como variável dependente. Isso foi possível porque o teste de Kolmogorov-Smirnov evidenciou normalidade da distribuição dessa variável na população estudada ( $\mathrm{p}=0,54)$. As variáveis que apresentaram $\mathrm{p}<0,20$ na análise simples pelo teste $\mathrm{H}_{\mathrm{o}}: \beta=0$ foram submetidas à regressão linear múltipla. Os resultados derivados dessa análise são apresentados neste artigo.

\section{Resultados}

Ano escolar, idade e sexo diferiram estatisticamente entre os adolescentes das escolas públicas e privadas (Tabela 1). Nas públicas, participou do estudo a maior proporção de adolescentes do 10 ano do ensino, e nas escolas privadas predominaram os alunos do 3o ano. Houve maior participação de adolescentes de 15 e 16 anos de idade, porém a proporção entre as faixas etárias foi mais equilibrada nas escolas privadas. Um fenômeno oposto foi observado em relação ao sexo, pois houve maior homogeneidade em relação ao sexo entre os alunos das escolas públicas. A religião mais referida pelos adolescentes foi a católica e a maioria coabitava com ambos os pais, não sendo observada diferença estatística significativa entre a religião e a coabitação segundo o tipo de escola.

Na Tabela 2, são descritas as fontes de informação sobre anticoncepção de emergência entre os adolescentes que relataram conhecer o método, independentemente do início da vida sexual, e as características do comportamento afetivo-sexual dos mesmos. Uma maior proporção de adolescentes tinha recebido informações sobre anticoncepção de emergência com amigos e conhecidos; seguida por escola e televisão. As fontes de conhecimento sobre anticoncepção de emergência menos referidas foram Internet, revistas ou jornais, serviços de saúde e farmácia. Não observou-se diferença estatisticamente significativa das fontes de informação sobre anticoncepção de emergência entre as escolas públicas e privadas. Em ambas as escolas, a maioria dos adolescentes não estava namorando, além disto, não havia iniciado a vida sexual e, portanto, não usou anticoncepção de emergência.

A Tabela 3 apresenta os percentuais de acerto para cada questão, segundo tipo de escola. A porcentagem de adolescentes que respondeu corretamente foi baixa na maioria das questões. Vale ressaltar que a proporção de acertos foi estatisticamente maior nas escolas privadas. Os itens com proporção mais elevada de acerto foram: a anticoncepção de emergência deve ser usada antes da relação sexual $(78,8 \%)$; a anticoncepção de emergência previne doenças sexualmente transmissíveis (DST) (75,3\%); e a anticoncepção de emergência é mais eficaz que outros métodos contraceptivos (54,5\%). Aqueles com menor proporção de acerto foram: ao usar a anticoncepção 
Características sociodemográficas dos adolescentes que conhecem a anticoncepção de emergência, por tipo de escola.

\begin{tabular}{|c|c|c|c|c|c|}
\hline \multirow[t]{2}{*}{ Variáveis } & \multicolumn{2}{|c|}{ Escola pública } & \multicolumn{2}{|c|}{ Escola privada } & \multirow[t]{2}{*}{ Valor de $p$ * } \\
\hline & $\mathrm{n}$ & $\%$ & $\mathrm{n}$ & $\%$ & \\
\hline Ano escolar & & & & & $<0,001$ \\
\hline 1o ano & 230 & 42,6 & 30 & 24,1 & \\
\hline 2o ano & 162 & 30,1 & 34 & 27,1 & \\
\hline 3으 ano & 147 & 27,3 & 61 & 48,8 & \\
\hline Idade (anos) & & & & & 0,005 \\
\hline $15-16$ & 347 & 64,5 & 63 & 50,6 & \\
\hline $17-19$ & 192 & 35,5 & 62 & 49,4 & \\
\hline Sexo & & & & & 0,019 \\
\hline Masculino & 259 & 48,0 & 45 & 36,1 & \\
\hline Feminino & 280 & 52,0 & 80 & 63,9 & \\
\hline Religião & & & & & 0,299 \\
\hline Católica & 218 & 40,4 & 42 & 33,7 & \\
\hline Evangélica & 177 & 32,9 & 51 & 40,9 & \\
\hline Nenhuma & 104 & 19,3 & 21 & 16,9 & \\
\hline Outras ** & 40 & 7,4 & 11 & 8,4 & \\
\hline Coabitação & & & & & 0,978 \\
\hline Ambos os pais & 405 & 75,1 & 95 & 75,9 & \\
\hline Somente mãe & 99 & 18,5 & 22 & 18,1 & \\
\hline Outras *** & 35 & 6,4 & 8 & 6,0 & \\
\hline Total & 539 & 100,0 & 125 & 100,0 & \\
\hline
\end{tabular}

* Nível descritivo do teste de associação pelo qui-quadrado;

** Outras religiões incluem: Espiritismo, Judaísmo, Testemunha de Jeová, Budismo e Islamismo;

*** Outras coabitações incluem: somente com o pai, amigos, avós e tios.

de emergência a mulher está protegida de uma gravidez até a chegada da menstruação $(26,1 \%)$; ao usar a anticoncepção de emergência, deve-se estar atento para que isto ocorra em até cinco dias $(24,5 \%)$; e em caso de vômito até 2 horas da ingestão da anticoncepção de emergência, devese repetir a dose $(15,7 \%)$.

As médias dos escores de conhecimento da anticoncepção de emergência, de acordo com as características sociodemográficas, perfil afetivosexual e fontes de informação sobre anticoncepção de emergência estão na Tabela 4. A análise univariada mostrou que os adolescentes que iniciaram a vida sexual e usaram anticoncepção de emergência tiveram uma média de acertos um pouco maior se comparados àqueles que não usaram $(\mathrm{p}<0,001)$. As variáveis que apresentaram $\mathrm{p}<0,20$ nessa análise foram: tipo de escola, idade, sexo, início da vida sexual/uso anterior da anticoncepção de emergência, e conhecer alguém que já usou a anticoncepção de emergência. As variáveis referentes à coabitação e fontes de informação, apesar de terem apresentado $\mathrm{p}<0,20$, não permaneceram na análise múltipla pelo fato de terem perdido força quando analisadas em conjunto com outras variáveis. Cabe destacar que as variáveis ano escolar e namoro também apresentaram $\mathrm{p}<0,20$, mas não permaneceram no modelo final devido à alta colinearidade com as variáveis idade e início da vida sexual, respectivamente. Esse resultado era esperado, uma vez que essas variáveis, segundo referenciais teóricos 3,15, "dizem" a mesma coisa. Não foram observadas variáveis de confusão, sendo o modelo final ajustado por algumas variáveis a fim de obter maior precisão do mesmo.

A análise de regressão linear múltipla, cujos resultados também estão descritos na Tabela 4, mostrou que o tipo de escola, ajustado por idade, sexo, início da vida sexual/uso anterior da anticoncepção de emergência e conhecer alguém que já utilizou a anticoncepção de emergência associou-se com o nível de conhecimento sobre anticoncepção de emergência. Alunos de escolas privadas apresentaram escore de conhecimento maior do que os de escolas públicas $(\beta=0,97$; IC95\%: -0,57; 1,37). Os resultados evidenciaram também que o escore de conhecimento foi 
Distribuição dos adolescentes segundo as fontes de informação sobre a anticoncepção de emergência e comportamento afetivo-sexual, por tipo de escola.

\begin{tabular}{|c|c|c|c|c|c|}
\hline \multirow[t]{2}{*}{ Variáveis } & \multicolumn{2}{|c|}{ Escola pública } & \multicolumn{2}{|c|}{ Escola privada } & \multirow[t]{2}{*}{ Valor de $p$ * } \\
\hline & $\mathbf{n}$ & $\%$ & $\mathbf{n}$ & $\%$ & \\
\hline \multicolumn{6}{|l|}{ Fontes de informação ** } \\
\hline Com amigos e conhecidos & 252 & 46,8 & 69 & 55,2 & 0,150 \\
\hline $\mathrm{Na}$ escola & 208 & 38,6 & 43 & 34,4 & 0,442 \\
\hline Na televisão & 184 & 34,1 & 38 & 30,4 & 0,488 \\
\hline Com pais e parentes & 145 & 26,9 & 27 & 21,6 & 0,269 \\
\hline Na Internet & 87 & 16,1 & 20 & 16,0 & 0,923 \\
\hline Em revistas e jornais & 79 & 14,7 & 22 & 17,6 & 0,492 \\
\hline Nos serviços de saúde & 67 & 12,4 & 21 & 16,8 & 0,249 \\
\hline Na farmácia & 34 & 6,3 & 07 & 5,6 & 0,928 \\
\hline \multicolumn{6}{|l|}{ Comportamento afetivo-sexual } \\
\hline Namoro atual & & & & & 0,008 \\
\hline Não & 341 & 68,5 & 95 & 57,2 & \\
\hline Sim & 157 & 31,5 & 71 & 42,8 & \\
\hline $\begin{array}{l}\text { Início da vida sexual/Uso anterior de anticoncepção de } \\
\text { emergência }\end{array}$ & \multicolumn{4}{|c|}{ emergência } & 0,032 \\
\hline Não iniciou a vida sexual/Não usou & 289 & 53,6 & 51 & 40,9 & \\
\hline Iniciou a vida sexual, mas não usou & 90 & 16,7 & 30 & 24,1 & \\
\hline Iniciou a vida sexual e usou & 134 & 24,9 & 40 & 32,0 & \\
\hline Não sabe se usou & 26 & 4,8 & 04 & 3,0 & \\
\hline $\begin{array}{l}\text { Conhece alguém que já usou anticoncepção de } \\
\text { emergência }\end{array}$ & & & & & 0,003 \\
\hline Não & 195 & 39,2 & 44 & 26,5 & \\
\hline Sim & 303 & 60,8 & 122 & 73,5 & \\
\hline Total & 498 & 100,0 & 166 & 100,0 & \\
\hline
\end{tabular}

* Nível descritivo do teste de associação pelo qui-quadrado;

** Questão que admitiu respostas múltiplas.

menor entre aqueles com menos idade, entre adolescentes do sexo masculino, aqueles que não tinham iniciado a vida sexual e não tinham usado anticoncepção de emergência antes, e entre os que não conheciam alguém que já tinha usado anticoncepção de emergência.

Assim, os fatores que determinaram um escore maior no conhecimento da anticoncepção de emergência foram: estudar em escola privada, ter idade mais avançada, ser do sexo feminino, ter iniciado a vida sexual e ter usado anticoncepção de emergência antes, e conhecer alguém que já usou anticoncepção de emergência.

\section{Discussão}

O conhecimento sobre anticoncepção de emergência foi maior nas escolas privadas do que nas públicas. Esse resultado ratifica que o nível de conhecimento sobre o tema está relacionado positivamente com o nível socioeconômico, sendo uma das variáveis mais importantes em sua definição 11, o que confirma nossa hipótese inicial. Entretanto, apesar dos alunos das escolas privadas terem alcançado maior proporção de acertos, os resultados mostraram que tanto um quanto o outro grupo de adolescentes sabe pouco sobre anticoncepção de emergência, o que está em acordo com a literatura 16,17.

É importante destacar, no entanto, que adolescentes das escolas públicas e privadas tinham perfis heterogêneos, sendo diferentes quanto ao ano escolar, idade, sexo e início da vida sexual/ uso anterior da anticoncepção de emergência, o que pode ter contribuído para a diferença do nível de conhecimento entre as escolas. Houve maior proporção de alunos do 1o ano das escolas públicas, que tinham mais turmas deste ano escolar se comparadas às turmas dos 2 o e 30 anos. 
Distribuição dos adolescentes que acertaram as questões de conhecimento sobre anticoncepção de emergência, por tipo de escola.

\begin{tabular}{|c|c|c|c|c|c|c|c|}
\hline \multirow[t]{2}{*}{ Questões } & \multicolumn{2}{|c|}{$\begin{array}{l}\text { Escola pública } \\
\qquad(n=539)\end{array}$} & \multicolumn{2}{|c|}{$\begin{array}{l}\text { Escola privada } \\
\qquad(n=125)\end{array}$} & \multicolumn{2}{|c|}{$\begin{array}{c}\text { Total } \\
(\mathrm{n}=664)\end{array}$} & \multirow[t]{2}{*}{ Valor de $p$ * } \\
\hline & $\mathrm{n}$ & $\%$ & $\mathrm{n}$ & $\%$ & $\mathbf{n}$ & $\%$ & \\
\hline $\begin{array}{l}\text { Anticoncepção de emergência deve ser usada antes da relação sexual } \\
\text { (resposta correta: falso) }\end{array}$ & 416 & 77,2 & 107 & 85,6 & 523 & 78,8 & 0,051 \\
\hline Anticoncepção de emergência previne DST (resposta correta: falso) & 392 & 72,7 & 108 & 86,4 & 500 & 75,3 & 0,002 \\
\hline $\begin{array}{l}\text { Anticoncepção de emergência é mais eficaz que outros métodos } \\
\text { contraceptivos (resposta correta: falso) }\end{array}$ & 276 & 51,2 & 86 & 68,8 & 362 & 54,5 & $<0,001$ \\
\hline $\begin{array}{l}\text { Anticoncepção de emergência precisa ser usada no período fértil } \\
\text { (resposta correta: falso) }\end{array}$ & 185 & 34,3 & 61 & 48,8 & 246 & 37,0 & 0,003 \\
\hline $\begin{array}{l}\text { Anticoncepção de emergência pode ser usada uma vez por mês } \\
\text { (resposta correta: falso) }\end{array}$ & 167 & 30,9 & 55 & 44,0 & 222 & 33,4 & 0,007 \\
\hline $\begin{array}{l}\text { Anticoncepção de emergência substitui a pílula oral comum (resposta } \\
\text { correta: falso) }\end{array}$ & 162 & 30,1 & 66 & 52,8 & 228 & 34,3 & $<0,001$ \\
\hline Anticoncepção de emergência é abortiva (resposta correta: falso) & 161 & 29,9 & 42 & 33,6 & 203 & 30,6 & 0,479 \\
\hline $\begin{array}{l}\text { Ao usar anticoncepção de emergência a mulher está protegida de } \\
\text { uma gravidez até a chegada da menstruação (resposta correta: falso) }\end{array}$ & 120 & 22,3 & 53 & 42,4 & 173 & 26,1 & $<0,001$ \\
\hline $\begin{array}{l}\text { Ao usar anticoncepção de emergência deve-se estar atento para que } \\
\text { isto ocorra em até } 5 \text { dias (resposta correta: verdadeiro) }\end{array}$ & 121 & 22,4 & 42 & 33,6 & 163 & 24,5 & 0,013 \\
\hline $\begin{array}{l}\text { Em caso de vômito até } 2 \text { horas da ingestão da anticoncepção de } \\
\text { emergência deve-se repetir a dose (resposta correta: verdadeiro) }\end{array}$ & 84 & 15,6 & 20 & 16,0 & 104 & 15,7 & 0,983 \\
\hline
\end{tabular}

DST: doenças sexualmente transmissíveis.

* Nível descritivo do teste de associação pelo qui-quadrado.

Além disso, apenas uma escola privada autorizou o desenvolvimento do estudo com estudantes do 1o ano, o que resultou em maior percentual de adolescentes das escolas públicas com 15 e 16 anos de idades. Dessa forma, há que se considerar que a maior proporção de alunos do 30 ano nas escolas privadas pode ter contribuído para o melhor resultado em relação ao nível de conhecimento, tendo em vista que a experiência sexual aumenta com a idade 3,18 .

De fato, a idade associou-se com maior nível de conhecimento sobre anticoncepção de emergência, assim como a experiência sexual, principalmente quando atrelada ao uso anterior do método, da mesma forma que constatado em outros estudos 10,19. Quanto mais velho o adolescente, maior a chance de já ter iniciado a vida sexual, o que o leva a se preocupar em obter informações sobre MAC, incluindo a anticoncepção de emergência, uma vez que passa a ser mais vulnerável a uma gravidez e pode se deparar com situações de descontinuidades e falhas no uso de métodos, justamente as indicações de seu uso 2 .

Em relação ao sexo, as meninas apresentaram média maior de escore de conhecimento em relação aos meninos, da mesma forma que em outro estudo ${ }^{8}$, o que é esperado tendo em vista ser um método de uso feminino. Adolescentes que conheciam alguém que já usou anticoncepção de emergência apresentaram maior nível de conhecimento. Provavelmente isso ocorre porque o uso da anticoncepção de emergência é comentado entre as amigas, compartilhamento que contribui para ampliar a divulgação e o conhecimento desse MAC 20, o que confirma o papel primordial que os pares exercem nas questões que cercam a contracepção na adolescência.

No que se refere à religião e coabitação, os adolescentes das escolas públicas e privadas se mostraram similares. Igualmente aos dados censitários de $2010^{21}$, a religião mais citada foi a católica, que apresenta especificidades quanto ao comportamento sexual e contraceptivo, pois alguns adeptos desta religião são menos flexíveis ao uso do preservativo masculino e à prática do sexo antes do casamento 22 , mas a religião não mostrou qualquer relação com o nível de conhecimento sobre anticoncepção de emergência.

Em relação às fontes de informação sobre anticoncepção de emergência, os amigos/conhecidos foi a mais referida pelos adolescentes, reiterando os resultados obtidos em outros 
Tabela 4

Análise de regressão linear simples e múltipla do nível do conhecimento da anticoncepção de emergência, segundo as características sociodemográficas, de comportamento afetivo-sexual e fontes de informação.

\begin{tabular}{|c|c|c|c|c|c|c|c|}
\hline \multirow[t]{2}{*}{ Variáveis } & \multicolumn{4}{|c|}{ Análise simples } & \multicolumn{3}{|c|}{ Análise múltipla } \\
\hline & Média (bruta) & DP & $\beta$ (bruta) & Valor de $p$ * & $\beta$ (ajustado) ** & IC95\% & Valor de $p$ *** \\
\hline \multicolumn{8}{|l|}{ Tipo de escola } \\
\hline Pública & 3,87 & 2,12 & 0,00 & - & - & - & - \\
\hline Privada & 5,14 & 2,00 & 1,27 & $<0,001$ & 0,97 & 0,$57 ; 1,37$ & $<0,001$ \\
\hline \multicolumn{8}{|l|}{ Ano escolar } \\
\hline 1 으 ano & 3,80 & 2,21 & 0,00 & - & - & - & - \\
\hline 2o ano & 3,86 & 2,03 & 0,09 & 0,656 & - & - & - \\
\hline 3o ano & 4,93 & 2,04 & 1,14 & $<0,001$ & - & - & - \\
\hline Idade & 4,18 & 2,16 & 0,33 & $<0,001$ & 0,19 & 0,$03 ; 0,35$ & 0,020 \\
\hline \multicolumn{8}{|l|}{ Sexo } \\
\hline Masculino & 3,81 & 2,25 & 0,00 & - & - & - & - \\
\hline Feminino & 4,50 & 2,04 & 0,71 & $<0,001$ & 0,48 & 0,$15 ; 0,81$ & 0,004 \\
\hline \multicolumn{8}{|l|}{ Coabitação } \\
\hline Ambos os pais & 4,11 & 2,18 & 0,00 & - & - & - & - \\
\hline Somente mãe & 4,54 & 2,11 & 0,43 & 0,049 & - & - & - \\
\hline Outras \# & 4,02 & 2,08 & $-0,11$ & 0,958 & - & - & - \\
\hline \multicolumn{8}{|l|}{ Namoro atual } \\
\hline Não & 4,05 & 2,09 & 0,00 & - & - & - & - \\
\hline $\operatorname{Sim}$ & 4,45 & 2,16 & 0,38 & 0,030 & - & - & - \\
\hline \multicolumn{8}{|l|}{ Conhece alguém que já usou } \\
\hline \multicolumn{8}{|l|}{ anticoncepção de emergência } \\
\hline Não & 3,55 & 0,14 & 0,00 & - & - & - & - \\
\hline $\operatorname{Sim}$ & 4,54 & 0,10 & 0,97 & $<0,001$ & 0,54 & 0,$19 ; 0,89$ & 0,003 \\
\hline \multicolumn{8}{|l|}{ Início da vida sexual/Uso anterior da } \\
\hline \multicolumn{8}{|l|}{ anticoncepção de emergência } \\
\hline Não iniciou vida sexual nem usou & 3,77 & 0,12 & 0,00 & - & 0,00 & - & - \\
\hline Iniciou a vida sexual, mas não usou & 4,55 & 0,19 & 0,72 & 0,001 & 0,57 & 0,$14 ; 1,00$ & 0,009 \\
\hline Iniciou a vida sexual e usou & 4,86 & 0,16 & 1,08 & $<0,001$ & 0,70 & 0,$31 ; 1,10$ & $<0,001$ \\
\hline Não sabe se usou & 3,31 & 0,39 & $-0,52$ & 0,193 & $-0,32$ & $-1,10 ; 0,46$ & 0,421 \\
\hline \multicolumn{8}{|l|}{ Fontes de informação da } \\
\hline \multicolumn{8}{|l|}{ anticoncepção de emergência } \\
\hline \multicolumn{8}{|l|}{ Pais/Parentes } \\
\hline Não & 4,11 & 2,20 & 0,00 & - & - & - & - \\
\hline $\operatorname{Sim}$ & 4,39 & 2,05 & 0,32 & 0,098 & - & - & - \\
\hline \multicolumn{8}{|l|}{ Amigos/Conhecidos } \\
\hline Não & 4,02 & 2,23 & 0,00 & - & - & - & - \\
\hline Sim & 4,36 & 2,08 & 0,36 & 0,032 & - & - & - \\
\hline \multicolumn{8}{|l|}{ Serviços de saúde } \\
\hline Não & 4,08 & 2,17 & 0,00 & - & - & - & - \\
\hline Sim & 4,89 & 1,95 & 0,79 & 0,001 & - & - & - \\
\hline \multicolumn{8}{|l|}{ Revistas ou jornais } \\
\hline Não & 4,12 & 2,14 & 0,00 & - & - & - & - \\
\hline Sim & 4,56 & 2,24 & 0,38 & 0,100 & - & - & - \\
\hline \multicolumn{8}{|l|}{ Farmácia } \\
\hline Não & 4,14 & 0,09 & 0,00 & - & - & - & - \\
\hline Sim & 4,90 & 0,34 & 0,81 & 0,020 & - & - & - \\
\hline \multicolumn{8}{|l|}{ Internet } \\
\hline Não & 4,13 & 2,19 & 0,00 & - & - & - & - \\
\hline Sim & 4,50 & 1,99 & 0,36 & 0,110 & - & - & - \\
\hline
\end{tabular}

DP: desvio-padrão; IC95\%: intervalo de 95\% de confiança.

* Teste de hipótese: $H_{0}: \beta_{1}=0$, testado para a heterogeneidade das médias:

** Ajustado por tipo de escola, idade, sexo, início da vida sexual/uso anterior da anticoncepção de emergência e conhece alguém que já usou a anticoncepção de emergência;

*** Teste de hipótese: $\mathrm{H}_{0}: \beta \mathrm{j}=0$;

\# Outras coabitações incluem: somente com pai, avós e tios. 
trabalhos 7,19. Apesar das distintas características entre os alunos, as fontes de informação não diferiram entre as escolas, ressaltando que os locais onde eles buscam informações e, principalmente o compartilhamento do conhecimento por meio do diálogo entre os pares, independem do ambiente escolar ao qual o aluno está inserido.

A Internet, apesar da sua relevância como meio de comunicação interativo e atrativo, amplamente disseminada na sociedade brasileira 23 , foi pouco mencionada. Esse resultado indica que quando se trata da busca por informações sobre sexualidade e contracepção, os adolescentes dão preferência aos amigos. Possivelmente, esse fenômeno ocorre pelo fato desse assunto ser de natureza pessoal e de foro íntimo, e os adolescentes se sentem mais seguros e menos expostos quando tiram suas dúvidas nas conversas entre amigos, certamente não se refere apenas a compartilhar informações técnicas, mas também suas vivências e experiências no uso da anticoncepção de emergência.

Curiosamente, para um quarto dos adolescentes a informação recebida ocorreu em casa por meio da família (pais e parentes). Aparentemente, algumas famílias atuam ativamente como fonte de informação acerca de sexualidade e contracepção. No entanto, há uma parcela de adolescentes que não procura os pais como um recurso de aprendizagem sobre métodos contraceptivos, revelando que as conversas sobre assuntos relativos a sexo são escassas, seja pela falta de informações adequadas dos pais ou pelo fato deste assunto ser considerado ainda um "tabu" no ambiente familiar 15 .

É preocupante o fato da baixa menção dos profissionais da saúde como fonte de informação sobre anticoncepção de emergência, porém há relatos de que os adolescentes têm pudor em buscar informações sobre sexualidade com adultos, e de que há certo despreparo dos profissionais para abordar a anticoncepção de emergência com este grupo 24.

Grande parte dos adolescentes revelou que nunca participou de oficinas sobre a anticoncepção de emergência nas escolas. De fato, quando questionadas, apenas uma escola comentou que oferecia casualmente atividades relacionadas ao tema para os alunos. Portanto, verifica-se que apesar de ter sido a segunda fonte de informação mais referida, aparentemente a escola é apenas o local onde ocorrem trocas de experiências entre os amigos, não atuando como instituição promotora de informações sobre anticoncepção de emergência. Estudo conduzido com adolescentes, professores e diretores de escolas públicas de São Paulo mostrou que a abordagem de temas sobre sexualidade e contracepção ainda ocorre de forma bastante incipiente nos ambientes escolares 25 , embora as aulas de educação sexual façam parte dos currículos escolares de São Paulo desde a década de 8026 .

A maioria dos adolescentes não havia iniciado a vida sexual e, consequentemente, não tinha utilizado a anticoncepção de emergência, diferentemente dos resultados de outros estudos 10,11. Esse resultado pode estar associado ao fato de que a maioria dos adolescentes era católica, conforme já discutido.

A análise das dez afirmativas específicas sobre o nível do conhecimento da anticoncepção de emergência revelou que as que obtiveram maior acerto em ambos os tipos de escolas foram: anticoncepção de emergência deve ser usada antes da relação sexual; anticoncepção de emergência previne DST, e anticoncepção de emergência é mais eficaz que os outros MAC. Estudo conduzido nos Estados Unidos 19 também concluiu que $69,6 \%$ das adolescentes assinalaram corretamente a anticoncepção de emergência como método contraceptivo pós-coital. Além disso, o nome popular "pílula do dia seguinte" sugere que o método deve ser usado após a relação sexual desprotegida. Provavelmente esses itens tiveram maior acerto porque estão em evidência nos debates entre amigos ou na mídia.

É importante destacar que os adolescentes não consideraram que a anticoncepção de emergência serve para prevenir as DST/AIDS, indicando que estão esclarecidos quanto a este aspecto. Vale lembrar que, com o surgimento da AIDS, houve ampla divulgação da importância do uso do preservativo masculino 27 . Além disso, as discussões sobre DST/AIDS estão comumente em pauta nas escolas, nos serviços de saúde e nas revistas em geral, de forma que isto pode ter contribuído para a elevada taxa de acertos nessa questão.

Os itens que apresentaram baixa proporção de acerto eram de natureza técnica e tinham um caráter mais específico, sobretudo em relação aos efeitos da anticoncepção de emergência em termos temporais e sua indicação de uso, os quais podem estar relacionados com a baixa obtenção de informação sobre anticoncepção de emergência nos serviços de saúde. Apesar de trabalhos realizados há quase uma década 28,29 apontarem os benefícios do uso da anticoncepção de emergência até 120 horas depois da relação sexual desprotegida, no Brasil até pouco tempo a indicação era que fosse ingerida até 72 horas, sendo esta recomendação ampliada para 120 horas 2 apenas recentemente. A alteração no tempo de uso, concomitantemente com o fato de que ainda há adolescentes que acreditam que a anticoncepção de emergência só deve ser usada no dia 
seguinte à relação sexual desprotegida, devido ao nome popular, contribui para os equívocos sobre o tempo de uso do método. Por último, salientase que muitos adolescentes acreditam, equivocadamente, que a anticoncepção de emergência protege a mulher durante todo o ciclo menstrual, ou seja, até a chegada da próxima menstruação.

A existência dessa confusão também foi observada em relação aos itens anticoncepção de emergência substitui a pílula oral comum, e anticoncepção de emergência pode ser usada uma vez por mês. Pode-se pensar que os adolescentes tendem a substituir a contracepção de uso regular, especialmente a anticoncepção hormonal oral pela anticoncepção de emergência, e que a utiliza rotineiramente, porém este fato não tem sido comprovado em trabalhos recentes 30,31 . De qualquer forma, essas são questões imprescindíveis que precisam ser esclarecidas, pois são dúvidas que podem acarretar o uso incorreto do método.

Em síntese, evidenciou-se que adolescentes do sexo feminino, com mais idade, do Ensino Médio das escolas privadas que já haviam iniciado a vida sexual, usado a anticoncepção de emergência e conheciam alguém que já usou o método, foram as que alcançaram maior escore de conhecimento da anticoncepção de emergência. Ademais, constatou-se que apesar dos adolescentes já terem ouvido falar sobre o método, poucos têm informações corretas sobre as indicações e mecanismos de ação da anticoncepção de emergência,e muitos têm ideias equivocadas sobre este recurso contraceptivo. A maioria dos adolescentes não teve informações sobre a anticoncepção de emergência nas escolas nem com profissionais de saúde. Esse resultado indicou que tanto a escola quanto o espaço social ou os serviços de saúde contribuem pouco para os conhecimentos sobre sexualidade e vida reprodutiva dos adolescentes. Nesse contexto, a experiência derivada deste estudo indica a necessidade de elaborar e executar novas propostas para educação em saúde reprodutiva nas escolas e nos serviços de saúde, que alcancem os adolescentes antes do início da vida sexual ou os preparem para agir quando ocorrem descontinuidades contraceptivas que os levem a optar pela anticoncepção de emergência.

Há que se destacar que o trabalho teve algumas limitações, dentre elas o acesso às escolas privadas. A maioria delas não autorizou a pesquisa alegando que a temática era imprópria aos adolescentes. As barreiras enfrentadas pela pesquisadora para acessar os alunos das escolas privadas ratificam a dificuldade em conduzir pesquisas neste tipo de instituição e, consequentemente, justificam a predominância de estudos sobre comportamento sexual e contraceptivo com alunos originários de escolas públicas. Na realidade, poucos são os trabalhos que possibilitam comparações entre adolescentes desses dois tipos de escolas e este é um ponto positivo dos resultados reportados neste artigo. 


\section{Resumen}

El objetivo de este estudio fue analizar el grado de conocimiento sobre la anticoncepción de emergencia en adolescentes de escuela secundaria en escuelas públicas y privadas. Se trata de un estudio transversal con la participación de 705 estudiantes, de 15 a 19 años, matriculados en educación secundaria en un municipio de São Paulo, Brasil. Se realizó un muestreo aleatorio estratificado por tipo de escuela y sistemático por clase. Cerca de un 24,9\% de los estudiantes de escuelas privadas y el $32 \%$ de las escuelas públicas habían iniciado su vida sexual, usando anticoncepción de emergencia. La puntuación media de los conocimientos fue 3,87 $(D P=2,12)$ en las escuelas públicas y 5,14 $(D P=2,00)$ en las privadas. El análisis de regresión lineal múltiple mostró que los adolescentes de las escuelas privadas, mujeres, mayores, que habian iniciado su vida sexual, utilizaban anticoncepción de emergencia y quienes utilizaron el método fueron los que lograron mejores resultados en conocimientos. Llegamos a la conclusión de que pocos adolescentes están informados sobre es método anticonceptivo.

Salud del Adolescente; Anticoncepción Postcoital; Salud Sexual y Reproductiva

\section{Colaboradores}

C. B. N. Chofakian e A. L. V. Borges contribuíram com a concepção do estudo, análise e interpretação dos dados e redação do artigo. E. Fujimori e L. A. K. Hoga contribuíram na interpretação dos dados e redação do artigo.

\section{Agradecimentos}

Projeto financiado pela Fundação de Amparo à Pesquisa do Estado de São Paulo (processo no 2010/14228-5).

\section{Referências}

1. World Health Organization. Emergency contraception. Report. WHO Technical Report Series, 244. http://www.who.int/mediacentre/factsheets/ fs244/en (acessado em 28/Ago/2012).

2. Ministério da Saúde. Anticoncepção de emergência: perguntas e respostas para profissionais de saúde. Brasília: Ministério da Saúde; 2011. (Série F. Comunicação e Educação em Saúde e direitos Sexuais e Direitos Reprodutivos - Caderno 3).

3. Borges ALV, Schor N. Início da vida sexual na adolescência e relações de gênero: um estudo transversal em São Paulo, Brasil, 2002. Cad Saúde Pública 2005; 21:499-507.
4. Ali MM, Cleland J. Sexual and reproductive behavior among single women aged 15-24 in eight Latin American countries: a comparative analysis. Soc Sci Med 2005; 60:1175-85.

5. Departamento de Ciência e Tecnologia, Secretaria da Ciência, Tecnologia e Insumos Estratégicos, Ministério da Saúde. PNDS 2006: Pesquisa Nacional de Demografia e Saúde da Criança e da Mulher: relatório. Brasília: Ministério da Saúde; 2008.

6. Mendonça RCM, Araújo TME. Métodos contraceptivos: a prática dos adolescentes das escolas agrícolas da Universidade Federal do Piauí. Esc Anna Nery Rev Enferm 2009; 13:863-71. 
7. Costa KBS, Carvalho ACG, Santos KB, Mendes SHFM, Soares A. Contracepção de adolescentes do Rio de Janeiro. Revista da SOGIA-BR 2006; 7:3-6.

8. Silva FC, Vitalle MSS, Maranhão HS, Canuto MHA, Pires MMS, Fisberg M. Diferenças regionais de conhecimento, opinião e uso de contraceptivo de emergência entre universitários brasileiros de cursos da área de saúde. Cad Saúde Pública 2010; 26:1821-31.

9. Cameron ST, Gordon R, Glasier A. The effect on use of making emergency contraception available free of charge. Contraception 2012; 86:366-96.

10. Araújo MSP, Costa LOBF. Comportamento sexual e contracepção de emergência entre adolescentes de escolas públicas de Pernambuco, Brasil. Cad Saúde Pública 2009; 25:551-62.

11. Perpétuo IHO. Contracepção de emergência na adolescência e a PNDS 2006. In: Arilha M, Lapa TS, Pisaneschi TC, organizadores. Contracepção de emergência no Brasil e América Latina: dinâmicas políticas e direitos sexuais e reprodutivos. São Paulo: Oficina Editorial; 2010. p. 91-120.

12. Paniz VMV, Fassa AG, Silva MC. Conhecimento sobre anticoncepcionais em uma população de 15 anos ou mais de uma cidade do Sul do Brasil. Cad Saúde Pública 2005; 21:1747-60.

13. Martins LBM, Costa-Paiva L, Osis MJD, Sousa MH, Neto AMP, Tadini V. Conhecimento sobre métodos anticoncepcionais por estudantes adolescentes. Rev Saúde Pública 2006; 40:57-64.

14. Borges ALV, Fujimori E, Hoga LAK, Contim M. Práticas contraceptivas entre jovens universitários: uso da anticoncepção de emergência. Cad Saúde Pública 2010; 26:816-26.

15. Pirotta KCM. Não há guarda-chuva contra o amor: estudo do comportamento reprodutivo e seu universo simbólico entre jovens universitários. Ciênc Saúde Coletiva 2002; 5:232-7.

16. Castro JF, Rodrigues VMCP. Conhecimentos e atitudes dos jovens face à contracepção de emergência. Rev Esc Enferm USP 2009; 43:889-94.

17. Adhikari R. Factors affecting awareness of emergency contraception among college students in Kathmandu, Nepal. BMC Womens Health 2009; 9:1-5.

18. Santelli JS, Lowry R, Brener N, Robin L. The association of sexual behavior with socioeconomic status, family structure and race/ethnicity among US adolescents. Am J Public Health 2000; 90:1582-8.

19. Ahern R, Frattarelli LA, Delto J, Kaneshiro B. Knowledge and awareness of emergency contraception in adolescentes. J Pediatr Adolesc Gynecol 2010; 23:273-8.
20. Fallon D. Accessing emergency contraception: the role of friends in the adolescent experience. Sociol Health Illn 2010; 32:677-94.

21. Instituto Brasileiro de Geografia e Estatística. Características gerais da população: resultados da amostra 2010. Rio de Janeiro: Instituto Brasileiro de Geografia e Estatística; 2010.

22. Silva CG, Santos AO, Licciardi DC, Paiva V. Religiosidade, juventude e sexualidade: entre a autonomia e a rigidez. Psicol Estud 2008; 13:683-92.

23. Instituto Brasileiro de Geografia e Estatística. Pesquisa nacional por amostra de domicílios 2009: síntese de indicadores. Rio de Janeiro: Instituto Brasileiro de Geografia e Estatística; 2010.

24. Nunes MT. Conhecimento e utilização da contracepção de emergência em alunas do ensino secundário em Guimarães. Revista Portuguesa de Clínica Geral 2005; 21:247-56.

25. Pirotta KCM, Barboza R, Pupo LR, Cavasin S, Unbehaum S. A educação sexual na escola: elementos para uma avaliação dos esforços realizados. BIS Bol Inst Saúde 2008; 46:34-7.

26. Castro MG, Abramovay M, Silva LB. Juventudes e sexualidade. Brasília: Organização das Nações Unidas para a Educação, Ciência e a Cultura; 2004.

27. Paiva V, Ayres JRCM, Segurado AC, Lacerda R, Silva NG, Silva MH, et al. A sexualidade de adolescentes vivendo com HIV: direitos e desafios para o cuidado. Ciênc Saúde Coletiva 2011; 16:4199-210.

28. Rodrigues I, Grou F, Joly J. Effectiveness of emergency contraceptive pills between 72 and 120 hours after unprotected sexual intercourse. Am J Obstet 2001; 184:531-7.

29. Ellertson C, Evans M, Ferden S, Leadbetter C, Spears A, Johnstone K, et al. Extending the time limit for starting the Yuzpe regimen of emergency contraception to 120 hours. Obstet Gynecol 2003; 101:1168-71.

30. Sidebottom A, Harrison PA, Donna A, Finnegan K. The varied circumstances prompting requests for emergency contraception at school-based clinics. J Sch Health 2008; 78:258-63.

31. Fine LC, Mollen CJ. A pilot study to assess candidacy for emergency contraception and interest in sexual health education in a pediatric emergency department population. Pediatr Emerg Care 2010; 26:413-6.

Recebido em 15/Ago/2013

Versão final reapresentada em 17/Dez/2013 Aprovado em 30/Jan/2014 